\title{
RESTRICTIONS OF SUBSPACES OF $C(X)\left({ }^{1}\right)$
}

\author{
BY \\ T. W. GAMELIN(2)
}

Introduction. Let $X$ be a compact Hausdorff space, $F$ a closed subset of $X, C(X)$ the Banach algebra of continuous complex-valued functions on $X$, and $A$ a closed linear subspace of $C(X)$. In this paper we describe when the restriction subspace $A \mid F$ is closed in $C(F)$, and what kind of extensions $g \in A$ of a function $f \in A \mid F$ we can hope to obtain, in terms of properties of measures orthogonal to $A$.

Theorem 1 gives necessary and sufficient conditions that $A \mid F$ be closed in $C(F)$, and necessary and sufficient conditions that $A \mid F$ be isometric with $A / k F$, $k F$ the space of functions in $A$ which vanish on $F$. In $\S$ III an extension constant $e(A \mid F)$ is associated with $A$ and $F$. Theorem 2 characterizes $e(A \mid F)$ in terms of induced mappings in the adjoint spaces. Theorems 2 and 3 combine to yield a generalized Rudin-Carleson theorem, which sharpens and extends a result of Bishop [1].

In $\S \mathrm{IV}, A$ is specialized to be a sup-norm algebra of functions on $X$, and the theory is applied to strengthen the interpolation theorems of Rudin [11] and Carleson [3] for analytic functions. $\S \mathrm{V}$ contains a discussion of intersections of peak sets in sup-norm algebras.

This paper is closely related to a paper of Glicksberg [6], and arose as an attempt to sharpen the methods and results of Glicksberg. The author is greatly indebted to this paper of Glicksberg, for the problems discussed there and the ideas found there.

The author would like to express his gratitude to Professor J. L. Kelley for initially expressing an interest in preliminary results and for valuable consultation and advice during the ensuing interim.

I. Fundamental lemmas. Let $M_{1}$ and $M_{2}$ be Banach spaces. Let $D$ be a closed linear subspace of $M_{1} \oplus M_{2}$, where the norm in $M_{1} \oplus M_{2}$ is defined by $\|a+b\|=\|a\|+\|b\|, a \in M_{1}, b \in M_{2}$. The null space of the natural map $M_{2} \rightarrow\left(M_{1} \oplus M_{2}\right) / D$ is $M_{2} \cap D$, and so the quotient map $U^{\prime}: M_{2} / M_{2} \cap D \rightarrow\left(M_{1} \oplus M_{2}\right) / D$ is norm-decreasing and 1-1. Let $P: D \rightarrow M_{1}$, $Q: M_{1} \rightarrow\left(M_{1} \oplus M_{2}\right) / D$, and $T: D \rightarrow M_{2} / M_{2} \cap D$ be the natural maps. Then the following diagram is anti-commutative.

Received by the editors April 10, 1963.

(1) This material is part of the author's Ph. D. thesis, submitted to the University of California at Berkeley, January, 1963.

(2) National Science Foundation Graduate Fellow. 


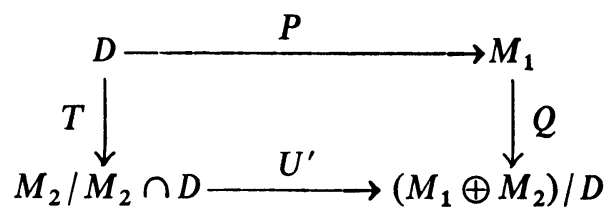

$D$ induces a closed subspace $S$ of $M_{1} \oplus\left(M_{2} / M_{2} \cap D\right)$. This subspace is the graph of an operator, also denoted by $S$, defined on the subspace $P(D)$ of $M_{1}$ with values in $M_{2} / M_{2} \cap D$. If $d=a+b, a \in M_{1}, b \in M_{2}$, then $S(a)$ $=b+M_{2} \cap D=T(d)=-\left(U^{\prime-1} \circ Q\right)(a)$.

LeMma 1. $S$ is bounded if and only if $U^{\prime-1}$ is bounded. If $U^{\prime} \neq 0$, i.e., if $M_{2}$ is not contained in $D$, then $\left\|U^{\prime-1}\right\|=\max (1,\|S\|) . U^{\prime}$ is an isometry if and only if $0 \leqq\|S\| \leqq 1$.

Proof. The case $U^{\prime}=0$ is trivial, so we assume $U^{\prime} \neq 0$.

Assume $U^{\prime-1}$ is bounded. Then $\|S\| \leqq\left\|U^{\prime-1}\right\|\|Q\| \leqq\left\|U^{\prime-1}\right\|$. Since $U^{\prime}$ is normdecreasing, $\left\|U^{\prime-1}\right\| \geqq 1$. Hence $\max (1,\|S\|) \leqq\left\|U^{\prime-1}\right\|$.

Conversely, assume $S$ is bounded. Let $r$ be a real number with $r>\max (1,\|S\|)$. If $c$ is in $M_{2}$, then $U^{\prime}\left(c+M_{2} \cap D\right)=c+D$. Let $d=a+b \in D, a \in M_{1}, b \in M_{2}$. Since $\left\|b+M_{2} \cap D\right\| \leqq\|S\|\|a\|$, there is an $e \in M_{2} \cap D$ such that $\|b+e\| \leqq r\|a\|$. Then

$$
\begin{aligned}
r\|c+d\| & =r\|c+a+b\|=r\|c+b\|+r\|a\| \\
& \geqq\|c-e\|-\|b+e\|+r\|a\| \geqq\|c-e\| \geqq\left\|c+M_{2} \cap D\right\| .
\end{aligned}
$$

Hence $\left\|U^{\prime-1}(c+D)\right\|=\left\|c+M_{2} \cap D\right\| \leqq r\|c+D\|$, and $\left\|U^{\prime-1}\right\| \leqq r$. Since $r>\max (1,\|S\|)$ was arbitrary, $\left\|U^{\prime-1}\right\| \leqq \max (1,\|S\|)$.

Combining the two inequalities, we see that $\left\|U^{\prime-1}\right\|=\max (1,\|S\|)$. The last statement of the lemma is an immediate consequence of this. Q.E.D.

Lemma 2. $S$ is bounded if and only if $0 \leqq\|T\|<1$. If $S$ is bounded, then $\|T\|=\|S\| /(1+\|S\|) . U^{\prime}$ is an isometry if and only if $0 \leqq\|T\| \leqq 1 / 2$.

Proof. Assume $S$ is bounded. Let $d=a+b \in D, a \in M_{1}, b \in M_{2}$. Then $\left\|b+M_{2} \cap D\right\| \leqq\|S\|\|a\|$ and $\|S\|\left\|b+M_{2} \cap D\right\| \leqq\|S\|\|b\|$, so $(1+\|S\|)\|T d\|=(1+\|S\|)\left\|b+M_{2} \cap D\right\| \leqq\|S\|(\|a\|+\|b\|)$ $=\|S\|\|d\|$. Hence $\|T\| \leqq\|S\| /(1+\|S\|)$. In particular, $0 \leqq\|T\|<1$.

Assume now $0<\|S\| \leqq \infty$. Let $r$ be a positive number with $r<\|S\|$. Choose $d=a+b \in D, a \in M_{1}, b \in M_{2}$, such that $r\|a\|<\left\|b+M_{2} \cap D\right\|=\|S a\|$. For $t>0$, choose $e \in M_{2} \cap D$ such that $\|b+e\|<\left\|b+M_{2} \cap D\right\|+t$. Let $c=d+e \in D$, then

$$
\begin{aligned}
\|c\| & =\|b+e\|+\|a\|<\left\|b+M_{2} \cap D\right\|+t+\left\|b+M_{2} \cap D\right\| / r \\
& =\|T c\|(1+r) / r+t .
\end{aligned}
$$




$$
\|T c\| /\|c\|>(1-t /\|c\|) r /(1+r) \geqq(1-t /\|a\|) r /(1+r) .
$$

Since $t>0$ was arbitrary, $\|T\| \geqq r /(1+r)$. If $\|S\|=\infty$, then $\|T\|=1$, while if $0<\|S\|<\infty$, then $\|T\| \geqq\|S\| /(1+\|S\|)$.

These facts can now be combined to complete the proof. Q.E.D.

II. The interpolation theorem. Let $A$ be a closed subspace of $C(X), X$ a compact Hausdorff space. Let $F$ be a closed subset of $X$, and let $A \mid F$ be the subspace of functions in $C(F)$ which are restrictions to $F$ of functions in $A$. The conjugate space of $C(X)$ may be identified with $M(X)$, the space of regular countably additive Borel measures on $X$. The space $M(F)$ of regular countably additive Borel measures on $F$ may be considered a subspace of $M(X)$. Conversely, every measure $m \in M(X)$ determines a restriction measure $m_{F} \in M(F)$, where $m_{F}(G)=m(F \cap G)$ for all Borel sets $G$.

The null space of the restriction map $U: A \rightarrow A \mid F$ is the space $k F$ of functions in $A$ which vanish on $F$. $U$ induces the quotient map $V: A|k F \rightarrow A| F$. The conjugate space of $A$ may be identified with $M(X) / A^{\perp}$, where $A^{\perp}$ is the set of measures in $M(X)$ which annihilate $A$. Similarly, the conjugate space of $A \mid F$ may be identified with $M(F) /(A \mid F)^{\perp}$, where $(A \mid F)^{\perp}=A^{\perp} \cap M(F)$. $U$ and $V$ induce the adjoint maps $U^{*}: M(F) /(A \mid F)^{\perp} \rightarrow M(X) / A^{\perp}$ and $V^{*}: M(F) /(A \mid F)^{\perp}$ $\rightarrow(A / k F)^{*}$.

LEMMA 3. The following statements are equivalent:

(i) $A \mid F$ is closed in $C(F)$.

(ii) Any one of the operators $V, V^{*}, U^{*}$ has a closed range.

(iii) Any one of the operators $V^{-1}, V^{*-1}, U^{*-1}$ is bounded.

If any of these statements hold, and $A \mid F \neq\{0\}$, then $\left\|V^{-1}\right\|=\left\|V^{*-1}\right\|$ $=\left\|U^{*-1}\right\| \geqq 1$. If the norms are equal to 1 , then $V, V^{*}$ and $U^{*}$ are isometries.

This lemma is an easy consequence of the fact that the range of an operator is closed if and only if the range of its adjoint is closed (cf. [5]). A version of Lemma 3 was used by Glicksberg [6]. The idea of passing to adjoint mappings to solve interpolation problems has been attributed to $\mathrm{E}$. Nelson, and makes an appearance in the book of Hoffman [9], where more references may be found.

The linear operator $T_{F}: A^{\perp} \rightarrow M(F) /(A \mid F)^{\perp}$ is defined by $T_{F}(m)=m_{F}+(A \mid F)^{\perp}$, $m \in A^{\perp}$. Also, if $m \in A^{\perp}$, we define $S_{F}\left(m_{F^{\prime}}\right)=m_{F}+(A \mid F)^{\perp}$. Now $M(X)$ $=M\left(F^{\prime}\right) \oplus M(F)$, where $\|m\|=\left\|m_{F}\right\|+\left\|m_{F^{\prime}}\right\|$ for any measure $m \in M(X)$. If the $M_{1}$ of $\S 1$ is taken to be $M\left(F^{\prime}\right), M_{2}$ is $M(F)$, and $D$ is $A^{\perp}$, then $S_{F}$ and $T_{F}$ are the operators $S$ and $T$ of Lemmas 1 and 2 , and $U^{*}$ is the operator $U^{\prime}$ of Lemma 1. Combining Lemmas 1, 2 and 3, we arrive at the following result.

Theorem 1. (i) $\left\|T_{F}\right\|<1$ if and only if $A \mid F$ is closed in $C(F)$.

(ii) $\left\|T_{F}\right\| \leqq 1 / 2$ if and only if $A \mid F$ is isometric with $A / k F$.

(iii) $\left\|T_{F}\right\|=\left\|S_{F}\right\| /\left(1+\left\|S_{F}\right\|\right)$.

(iv) If $A \mid F \neq\{0\}$, then $\left\|U^{*^{-1}}\right\|=\max \left(1,\left\|S_{F}\right\|\right)=\left\|V^{-1}\right\|$. 
COROLlary. (i) $A \mid F=C(F)$ if and only if there is a constant $t<1$ such that $\left\|m_{F}\right\| \leqq t\|m\|$ for all measures $m \in A^{\perp}$.

(ii) $A / k F$ is isometric with $C(F)$ if and only if $\left\|m_{F}\right\| \leqq\|m\| / 2$ for all measures $m \in A^{\perp}$.

Proof. If $A \mid F=C(F)$, then $(A \mid F)^{\perp}=\{0\}$ and $T_{F}(m)=m_{F}$. The result then follows from Theorem 1. Conversely, if $\left\|m_{F}\right\| \leqq t\|m\|$ for some $t<1$ and all $m \in A^{\perp}$, then $(A \mid F)^{\perp}=\{0\}$, and $\left\|T_{F}\right\| \leqq t$. Again Theorem 1 applies. Q.E.D.

EXAMPLE. Let $A$ be the algebra of functions continuous on the unit disc $|z| \leqq 1$ in the complex plane, and analytic in the interior of the disc. For a fixed $r \geqq 0$, let $B_{r}$ be the linear subspace of $A$ of functions which satisfy $f(0)=r f(1)$, and let $F=\{1\}$. Every measure $n \in B_{r}^{\perp}$ is of the form $n=n^{\prime}+t r m_{1}-t m_{0}$, where $n^{\prime} \in A^{\perp}$ and $m_{z}$ is the unit point mass at $z$. Now $\left\|m_{0}+A^{\perp}\right\| \geqq\left|\left(m_{0}+A^{\perp}\right)(1)\right|=\left|m_{0}(1)\right|=1$, so $\|n\|=\left\|n^{\prime}+t r m_{1}-t m_{0}\right\|=|t| r+\left\|n^{\prime}-t m_{0}\right\| \geqq|t| r+|t|$. Hence $\left\|n_{F}\right\| /\|n\| \leqq|t| r /[|t| r+|t|]=r /(1+r)$, and $\left\|T_{F}\right\| \leqq r /(1+r)$. Taking $n=r m_{1}-m_{0}$ above, we see that $\left\|T_{F}\right\|=r /(1+r)$, and so $\left\|S_{F}\right\|=r$. In particular, $\left\|S_{F}\right\|$ may assume any value between 0 and $\infty$.

III. The extension constant. In the preceding example, the number $r$ is an indicator of how well we can expect to extend functions $f \in B_{r} \mid F$ to functions $g \in B_{r}$. If $r>1$, functions $g \in B_{r}$ satisfying $g(1)=1$ must have an average value of $r$ on the circle $|z|=1$, and so $\|g\|>r$. Similarly, if $r \leqq 1$, functions $g \in B_{r}$ satisfying $g(1)=1=\|g\|$ cannot satisfy $|g(z)|<r$ on too much of the unit circle. Except for these restrictions, it should be possible to extend the function $f(1)=1$ more or less as we please. In the following, we define an extension constant as a measure of how well we can hope to interpolate functions in restriction subspaces, and then characterize this constant in terms of the maps induced in the adjoint spaces.

Let $A$ be a closed subspace of $C(X), F$ a closed subset of $X$, and $t>0 . A \mid F$ is said to have property $E_{r}$ if the following condition holds: given $f \in A \mid F$ with $\|f\|<1$ and a closed subset $G$ of $X$ disjoint from $F$, there exists an extension $g \in A$ of $f$ such that $\|g\|<\max (1, t)$ and $|g(x)|<t$ for all $x \in G$. The extension constant $e(A \mid F)$ associated with $A \mid F$ is defined to be

$$
e(A \mid F)=\inf \left\{t: A \mid F \text { has property } E_{t}\right\} .
$$

If $A \mid F$ has property $E_{t}$ for no $t$, then we define $e(A \mid F)=\infty$.

THEOREM 2. Let $A$ be a closed subspace of $C(X)$, and let $F$ be a closed subset of $X$. Let $S_{F}: m_{F^{\prime}} \rightarrow m_{F}+(A \mid F)^{\perp}$ and $T_{F}: m \rightarrow m_{F}+(A \mid F)^{\perp}$ be the linear operators of Theorem 1 , defined for $m \in A^{\perp}$. Then

$$
e(A \mid F)=\left\|S_{F}\right\|=\left\|T_{F}\right\| /\left(1-\left\|T_{F}\right\|\right) \text {. }
$$

Proof. Assume $\left\|S_{F}\right\|>e(A \mid F)$. Choose $m \in A^{\perp}$ such that $\left\|m_{F^{\prime}}\right\|=1$ and 
$\left\|m_{F}+(A \mid F)^{\perp}\right\|>e(A \mid F)$. Choose $f \in A \mid F$ such that $\|f\|<1$ and $\int_{F} f d m=r>e(A \mid F)$. Choose a closed subset $G$ of $X$, disjoint from $F$, such that the variation of $m$ on $(F \cup G)^{\prime}$ is less than $[r-e(A \mid F)][\max (1, e(A \mid F))]^{-1}$. Let $g \in A$ be an extension of $f$ satisfying $\|g\|<\max (1, e(A \mid F))$ and $|g(x)|<e(A \mid F)$ for $x \in G$. Then

$$
\begin{aligned}
0=\left|\int_{\boldsymbol{X}} g d m\right| & \geqq\left|\int_{\boldsymbol{F}} g d m\right|-\left|\int_{G} g d m\right|-\left|\int_{(F \cup G)^{\prime}} g d m\right| \\
& \geqq r-e(A \mid F)-\|g\| \int_{(F \cup G)^{\prime}} d|m|>0 .
\end{aligned}
$$

From this contradiction, it follows that $\left\|S_{F}\right\| \leqq e(A \mid F)$.

Now assume $\left\|S_{F}\right\| \geqq 1$. By Theorem $1,\left\|S_{F}\right\|=\left\|V^{-1}\right\|$. If $f \in A \mid F,\|f\|<1$, then there is an extension $g \in A$ of $f$ satisfying $\|g\|<\left\|V^{-1}\right\|$. Thus $e(A \mid F)$ $\leqq\left\|S_{F}\right\|$, and so $e(A \mid F)=\left\|S_{F}\right\|$.

Finally, assume $0 \leqq\left\|S_{F}\right\|<1$. Let $p$ be a positive continuous function satisfying $\left\|S_{F}\right\|<p(x) \leqq 1$. Let $B=\{g: p g \in A\}$. B is a closed linear subspace of $C(X)$, $B^{\perp}=\left\{p m: m \in A^{\perp}\right\}, B \mid F=\{f: p f \in A \mid F\}$, and $(B \mid F)^{\perp}=\left\{p m: m \in(A \mid F)^{\perp}\right\}$. If $p m \in B^{\perp}$, then

$$
\begin{aligned}
\left\|(p m)_{F}+(B \mid F)^{\perp}\right\| & \leqq\left\|m_{F}+(A \mid F)^{\perp}\right\| \leqq\left\|S_{F}\right\|\left\|m_{F^{\prime}}\right\| \\
& \leqq\left\|S_{F}\right\|[\inf p(x)]^{-1} \int_{F^{\prime}} p d|m|=\left\|S_{F}\right\|[\inf p(x)]^{-1}\left\|(p m)_{F}{ }^{\prime}\right\| .
\end{aligned}
$$

Hence the operator $S_{F}^{\prime}: n_{F^{\prime}} \rightarrow n_{F}+(B \mid F)^{\perp}$, defined for $n \in B^{\perp}$ has norm $\left\|S_{F}^{\prime}\right\|<1$. Let $f \in A \mid F,\|f\|<1$, and let $G$ be a closed subset of $X$ disjoint from $F$. Choose $p$ so that $p \mid F=1$ and $p$ is near to $\left\|S_{F}\right\|$ on $G$. As in the preceeding paragraph, we can find an extension $p^{-1} g \in B$ of $p^{-1} f=f$ such that $\left\|p^{-1} g\right\|<1$. $g \in A$ is an extension of $f$ satisfying $|g(x)|<p(x)$ for all $x \in X$. In particular, $\|g\|<1$, and $|g(x)|$ cannot be too much larger than $\left\|S_{F}\right\|$ on $G$. Hence $A \mid F$ has property $E_{t}$ for all $t>\left\|S_{F}\right\|$. By definition, $e(A \mid F) \leqq\left\|S_{F}\right\|$. Hence $e(A \mid F)=\left\|S_{F}\right\|$. Q.E.D.

The " $1 / 22^{n}$-argument," originally introduced by Bishop for problems of this type, allows us to strengthen a part of the theorem above. In fact, the introduction of the positive function $p$ is also due to Bishop [1].

THeOREM 3. Let $A$ be a closed subspace of $C(X)$. Let $F$ be a closed subset of $X$ with extension constant $e(A \mid F)<1$. Let $f \in A \mid F$ satisfy $\|f\|=1$. Let $p$ be a continuous positive function on $X$ such that $|f(x)| \leqq p(x)$ for all $x \in F$, and $p(x)>e(A \mid F)$ for all $x \in X$. Then there is an extension $g \in A$ of $f$ such that $|g(x)| \leqq p(x)$ for all $x \in X$.

Proof. We may assume $p(x) \leqq 1$ for $x \in X$. Let $B=p^{-1} A$, as in the proof of Theorem 2 . The same estimates as used there yield the inequality

$$
e(B \mid F) \leqq e(A \mid F)[\inf p(x)]^{-1}<1 .
$$


If $p^{-1} f \in B \mid F$ has a norm-preserving extension $p^{-1} g \in B$, then $g$ is the required extension of $f$. Thus it suffices to show that, under the assumption $e(A \mid F)<1$, every $f \in A \mid F$ can be extended to $g \in A$ satisfying $\|g\|=\|f\|$.

Let $e(A \mid F)<s<1$, and define $t_{n}=(1-s) / 2^{n+1}$. Let $h_{1} \in A$ be an extension of $f$ satisfying $\left\|h_{1}\right\| \leqq 1+t_{1}$. Assume $h_{j}$ has been chosen for $1 \leqq j \leqq n$. Let $W_{n}=\left\{x:\left|h_{j}(x)\right| \geqq 1+t_{n+1}\right.$ for some $\left.j \leqq n\right\}$. Let $h_{n+1} \in A$ be an extension of $f$ such that $\left\|h_{n+1}\right\| \leqq 1+t_{n+1}$ and $\left|h_{n+1}(x)\right|<s$ for $x \in W_{n}$. Let $g=\sum_{j=1}^{\infty} h_{j} / 2^{j}$; then $g \in A$ is an extension of $f$. If $x \notin W_{n}$ for all $n$, then $\left|h_{j}(x)\right| \leqq 1$ for all $j$, so $|g(x)| \leqq 1$. Assume $x \in W_{n}-W_{n-1}$. Then $\left|h_{j}(x)\right|<1+t_{n}$ for $1 \leqq j \leqq n$, and $\left|h_{j}(x)\right|<s$ for $j \geqq n+1$. So

$$
\begin{aligned}
|g(x)| & \leqq \sum_{j=1}^{\infty}\left|h_{j}(x)\right| / 2^{j} \leqq\left(1+t_{n}\right) \sum_{j=1}^{n} 1 / 2^{j}+\sum_{j=n+1}^{\infty} s / 2^{j} \\
& \leqq\left(1+t_{n}\right)\left(1-1 / 2^{n}\right)+s / 2^{n} \\
& \leqq 1+(1-s) / 2^{n+1}-1 / 2^{n}-(1-s) / 2^{2 n+1}+s / 2^{n} \\
& \leqq 1+(1-s)\left[1 / 2^{n+1}-1 / 2^{2 n+1}-1 / 2^{n}\right]<1 .
\end{aligned}
$$

Thus $\|g\| \leqq 1$. So $\|g\|=\|f\|=1$. Q.E.D.

A subset $F$ of $X$ is said to be a peak set of the subspace $A$ of $C(X)$ if there is a function $f \in A$ satisfying $f^{-1}(1)=F$ and $|f(x)|<1$ for $x \notin F$. The function $f$ satisfying these conditions is said to peak on $F$.

CoRollary. Let $A$ be a closed subspace of $C(X)$, and let $F$ be a closed subset of $X$. If $e(A \mid F)<1$ and $A \mid F$ contains the constant functions, then $F$ is an intersection of peak sets.

EXAMPLE. (i) Let $A$ be a closed separating subalgebra of $C(X)$ which contains the constant functions. Suppose the Silov boundary $b(A)$ of $A$ is a proper subset of $X$. Then $e(A \mid b(A))=1$, while every function $f \in A \mid b(A)$ has a norm-preserving extension $g \in A$.

(ii) Let $B_{1}$ be the subalgebra of $C(|z| \leqq 1)$ discussed in $\S$ II, and let $F=\{1,-1\}$. Then $e(A \mid F)=1$. If $h \in C(F)$ is defined by $h(1)=1, h(-1)=-1$, then it is easy to see that $h$ has no norm-preserving extensions $g \in B_{1}$. This example is due to Glicksberg [6].

IV. Sup-norm algebras. A sup-norm algebra is a closed subalgebra of $C(X)$ which separates points and contains the constant functions. Subsets of $X$ which are intersections of peak sets seem to play an important role for sup-norm algebras. In the following, we study the extension constant $e(A \mid F)$ for $A$ a sup-norm algebra, and relate it to intersections of peak sets.

THEOREM 4. Let $A$ be a sup-norm algebra on $X$, and let $F$ be a closed subset of $X$. Then either $e(A \mid F)=0$ or $e(A \mid F) \geqq 1$. 
Proof. Suppose $e(A \mid F)<1$. Let $G$ be a closed subset of $X$ disjoint from $F$. Let $f \in A \mid F$ satisfy $\|f\|<1$, and let $g \in A$ be an extension of $f$ satisfying $\|g\|<1$. Let $h \in A$ be an extension of the constant function $1 \in A \mid F$ such that $\|h\|=1$ and $|h(x)|<1$ for $x \in G$. Then $h^{n} g \in A$ is also an extension of $f, h^{n} g$ is small on $G$ if $n$ is large, and $\left\|h^{n} g\right\| \leqq\|g\|<1$. Thus $e(A \mid F)=0$. Q.E.D.

To prove the next theorem we need the following result of Glicksberg [6]. It is proved by proper modification of the Bishop " $1 / 2^{n}$-argument" (cf. Theorem 3).

Lemma 4. Let $A$ be a closed subalgebra of $C(X)$, and let $F$ be an intersection of peak sets of $A$. Then any $f \in A \mid F$ has an extension $g \in A$ satisfying $\|g\|=\|f\|$.

THEOREM 5. Let $A$ be a closed subalgebra of $C(X)$, and let $F$ be an intersection of peak sets of $A$. Then $e(A \mid F)=0$.

Proof. Let $G$ be a closed subset of $X$ disjoint from $F$. Let $f \in A \mid F$ satisfy $\|f\|=1$, and let $g \in A$ be a norm-preserving extension of $f$. Choose $h \in A$ to peak on a set $H$ containing $F, H$ disjoint from $G . h^{n} g \in A$ is also a norm-preserving extension of $f$, and $h^{n} g$ is small on $G$ when $n$ is large. Hence $e(A \mid F)=0$. Q.E.D.

COROLlary. Let $A$ be a sup-norm algebra on $X$, and let $F$ be a closed subset of $X . F$ is an intersection of peak sets if and only if $e(A \mid F)=0$. This occurs if and only if $m_{F} \in A^{\perp}$ for all measures $m \in A^{\perp}$.

This corollary is the result of combining the preceding theorem with Theorem 2 and the corollary to Theorem 3. It should be noted that Glicksberg [6] proved that if $m_{F} \in A^{\perp}$ for all measures $m \in A^{\perp}$, then $F$ is an intersection of peak sets.

EXAMPLES. (i) A logmodular algebra is a sup-norm algebra for which the logarithms of the moduli of the invertible elements in the algebra are dense in $C_{R}(X)$, the space of continuous real-valued functions on $X$. A sup-norm algebra $A$ is logmodular if and only if every positive function in $C(X)$ can be approximated uniformly by a sequence $\left\{\left|f_{n}\right|\right\}$, where $f_{n} \in A$ and $1 / f_{n} \in A$. In particular, Dirichlet algebras are logmodular (cf. [8]). Glicksberg [6] has shown that if $A$ is logmodular on $X$, and if $A \mid F$ is closed in $C(F)$ for the closed subset $F$ of $X$, then $m \in A^{\perp}$ implies $m_{F} \in A^{\perp}$. In other words, the following result obtains.

THEOREM. If $A$ is a logmodular algebra on $X$, and $F$ is a closed subset of $X$, then either $e(A \mid F)=0$ or $e(A \mid F)=\infty$. In particular, every point $x \in X$ is an intersection of peak sets.

(ii) The preceding results enable us to state a strengthened form of the RudinCarleson theorem (cf. $[3 ; 11]$ ).

THEOREM. Let $F$ be a closed subset of the boundary of the unit disc $|z| \leqq 1$ in the complex plane. Assume $F$ has one-dimensional Lebesgue measure zero. Let $f \in C(F)$, and let $p$ be a positive continuous function on $|z| \leqq 1$ such that 
$|f(x)| \leqq p(x)$ for $x \in F$. Then there is a function $g \in C(|z| \leqq 1)$ which is analytic in $|z|<1$, which extends $f$, and which satisfies $|g(z)| \leqq p(z)$ for all $|z| \leqq 1$. Moreover, if $\left|z_{j}\right|<1,1 \leqq j \leqq k$, and if $n_{j}$ are positive integers, $1 \leqq j \leqq k$, then $g$ can be chosen to have a zero at $z_{j}$ of order at least $n_{j}$.

Proof. Let $A$ be the subalgebra of $C(|z| \leqq 1)$ of functions which are analytic in $|z|<1$ and which have zeros at $z_{j}$ of order at least $n_{j}, 1 \leqq j \leqq k$. Let $B$ be the restriction of $A$ to the circle $|z|=1$. From the F. and M. Riesz theorem we conclude that $m_{F}=0$ for every measure $m \in B$. By Theorem $1, B \mid F=C(F)$. By Theorem 2, $e(B \mid F)=0$. By Theorem 3, there is an $h \in A$ such that $h(F)=1$ and $|h(z)|<1$ for all $z \notin F$ satisfying $|z|=1$. Hence $|h(z)|<1$ for all $|z| \leqq 1$, $z \notin F$. $F$ is a peak set of $A$, so by Theorem $5 e(A \mid F)=0$. The result then follows from Theorem 3. Q.E.D.

$V$. Intersections of peak sets. This section contains a discussion of intersection of peak sets of a sup-norm algebra $A$ on $X$. The Šilov boundary of $A$ will be denoted by $b(A)$. A point $x \in X$ is said to be a strong boundary point of $A$ if $x$ is an intersection of peak sets of $A$ (cf. [10]). The collection of strong boundary points of $A$ is the strong boundary of $A$, and is denoted by $b^{\prime}(A)$. Clearly, $b^{\prime}(A)$ is contained in $b(A)$.

The following lemma was used crucially by Rickart [10] to prove that the strong boundary of $A$ is actually a boundary for $A$, i.e., every function $f \in A$ assumes its maximum modulus on $b^{\prime}(A)$. In fact, every function $f \in A$ assumes its maximum modulus on some peak set, and the following lemma, together with Zorn's lemma, shows that every peak set contains a strong boundary point.

Lemma 5 (CF. [10, Lemma 3. 3.12]). Let $A$ be a sup-norm algebra on $X$. Suppose $F$ is an intersection of peak sets of $A$, and $G \subset F$ is an intersection of peak sets of $A \mid F$. Then $G$ is an intersection of peak sets of $A$.

Proof. If $m \in A^{\perp}$, then $m_{F} \in(A \mid F)^{\perp}$, so $m_{G} \in(A \mid G)^{\perp}$. The result follows from the corollary to Theorem 5. Q.E.D.

This lemma can also be used to obtain the following result, which is equivalent to the Šilov idempotent theorem for sup-norm algebras (cf. [10]).

Lemma 6. Let $A$ be a sup-norm algebra on $X$, and assume $X$ is the maximal ideal space of $A$. Let $F$ be an intersection of peak sets of $A$. Then any closed and open subset of $F$ is an intersection of peak sets of $A$. Any component of $F$ is an intersection of peak sets of $A$.

Proof. The maximal ideal space of $A \mid F$ is the set of points $y \in X$ satisfying $|f(y)| \leqq\|f \mid F\|$ for all $f \in A$. This set is precisely $F$, since any such $y$ belongs to every peak set which contains $F$. From the Šilov idempotent theorem, it follows that any closed and open subset of $F$ is a peak set of $A \mid F$. Also, any component of $F$ is an intersection of peak sets of $A \mid F$. The result then follows from the preceeding lemma. Q.E.D. 
LEMMA 7. Let $A$ be a sup-norm algebra on $X$, and let $F$ and $G$ be intersections of peak sets of $A$. Then $F \cup G$ is an intersection of peak sets of $A$.

Proof. $F \cap G$ is an intersection of peak sets of $A$. Consequently, $m \in A^{\perp}$ implies $m_{F \cup G}=m_{F}+m_{G}-m_{F \cap G} \in A^{\perp}$. By the corollary to Theorem 5, $F \cup G$ is an intersection of peak sets. Q.E.D.

Any intersection of intersections of peak sets is again an intersection of peak sets. Hence the subsets of $X$ which are intersections of peak sets are the closed sets of a topology for $X$, which we call the extension topology or e-topology for $X$. An open subset $W$ of $X$ is e-open if and only if $W^{\prime}$ is an intersection of peak sets, i.e., if and only if $e\left(A \mid W^{\prime}\right)=0$.

If $F$ is an intersection of peak sets, then $F$ is a hull. Hence the $e$-topology for $X$ is smaller than the hull-kernel topology for $X$, which, in turn, is smaller than the given topology for $X$.

The $e$-topology is a $T_{1}$-topology if and only if every point in $X$ is a strong boundary point of $A$. A conjecture of Bishop can be stated as follows: If $X$ is the maximal ideal space of $A$, and if the $e$-topology is a $T_{1}$-topology, then $A=C(X)$. From a result of Glicksberg [7], it follows that the e-topology is a Hausdorff topology if and only if $A=C(X)$.

Remarks. The corollary to Theorem 5 is essentially due to Glicksberg. Lemma 7 is due to H. S. Bear, and this proof is given by Glicksberg. (Cf. Remarks, [6].)

\section{BiBLIOGRAPHY}

1. E. Bishop, A general Rudin-Carleson theorem, Proc. Amer. Math. Soc. 13(1962), 140-143.

2. - A generalization of the Stone-Weierstrass theorem, Pacific J. Math. 11(1961), 777-784.

3. L. Carleson, Representations of continuous functions, Math. Z. 66 (1957), 447-451.

4. L. de Branges, The Stone-Weierstrass theorem, Proc. Amer. Math. Soc. 10 (1959), 822-824.

5. N. Dunford and J. Schwartz, Linear operalors. Part I, Interscience, New York, 1958.

6. I. Glicksberg, Measures orthogonal to a gebras and sets of antisymmetry, Trans. Amer. Math. Soc. 105(1962), 415-435.

7. - Function algebras with closed restrictions, Proc. Amer. Math. Soc. 14(1963), $158-161$.

8. K. Hoffman, Analytic functions and logmodular Banach algebras, Acta Math. 108(1962), 271-317.

9. —_ Banach spaces of analytic functions, Prentice-Hall, Englewood Cliffs, N. J, 1961.

10. C. Rickart, General theory of Banach algebras, Van Nostrand, New York, 1960.

11. W. Rudin, Boundary values of continuous analytic functions, Proc. Amer. Math. Soc. 7 (1956), 808-811.

12. G. Silov, On rings of functions with uniform convergence, Ukrain. Mat. Ž. 3(1951), 404-411.

IUniversity of CALIFornia, BERKELEY,' CALIFORNIA 